\title{
O COORDENADOR E A HIDROCEFALIA: UM ELO ENTRE O ATENDIMENTO EDUCACIONAL ESPECIALIZADO, DOCENTES, COORDENADORES E A EQUIPE DE SAÚDE
}

\author{
Luiz Flávio da Silva \\ Rosângela Maria Marinho de Melo \\ Alexandro Lucas da Silva \\ Allison José dos Santos \\ Leandro Rafael Cunha de Oliveira \\ Prof. Dr. Diogenes José Gusmão Coutinho
}

RESUMO: O presente trabalho objetivou analisar a importância do coordenador e a hidrocefalia: um elo entre o atendimento educacional especializado, docentes, coordenadores e a equipe de saúde. A palavra hidrocefalia se define como dilatação das cavidades ventriculares cerebrais, ocasionado por um aumento de volume da pressão do líquido céfalo raquidiano (LCR). Ocorre o crescimento excessivo do perímetro cefálico, decorrendo em fontanelas tensas, vômitos, cefaléia, déficits cognitivos e visuais. $\mathrm{O}$ procedimento cirúrgico consiste na introdução de um dreno, sendo mais utilizado ventrículo-peritoneal, onde os riscos de infecção estão ressaltados. A fisioterapia dever ser realizada freqüentemente, com objetivo de ajudar na habilidade e movimento da criança. A assistência prestada à criança portadora desta doença deve ser realizada com sólido conhecimento técnico-científico, para que as ações sejam praticadas de forma segura. Considerando a importância da assistência do coordenador, docente, AEE, enfermeiro e outros profissionais neste caso da área de saúde frente a está patologia, objetivou-se, com o presente estudo, descrever as ações do coordenador, docente, $\mathrm{AEE}$, enfermeiro e outros profissionais durante a assistência ao portador de hidroceefalia. Neste contexto, cabe ao coordenador, docente, AEE, enfermeiro e outros profissinais elaborar e implementar estratégia que venha melhorar não somente o quadro clínico da doença, bem como promover a saúde e adaptação dessas crianças. Esta pesquisa tratar-se de um trabalho de revisão bibliográfica.

Descritores: Hidrocefalia. Pedagogo. Pediátrico. Cuidados de docentes.

ABSTRACT: The word hydrocephalus is defined as dilation of cerebral ventricles, caused by an increase in volume of the pressure of the cerebrospinal fluid (CSF). Is the excessive growth of head circumference, happening in tense fontanelle, vomiting, headache, cognitive and visual deficits. The surgical procedure is the introduction of a drain, being used ventriculo-peritoneal where the infection risks are underlined. Physical therapy should be carried out regularly, in order to assist in the child's ability and movement. The care provided to children living with this disease should be performed with solid technical and scientific knowledge, that the actions are carried safely. Considering the importance of the nurses' assistance is pathology, the aim, with this study, describe the actions of the nurse for assistance to hidroceefalia carrier. In this context, it is the nurse develop and implement strategy 
that will not only improve the clinical picture of the disease and promote health and adaptation of these children. This research deal is a bibliographical review.

Keywords: Education. Pediatric nurse. Research.

\section{INTRODUÇÃO}

A Hidrocefalia é uma condição clínica que acomete o cliente por toda vida e se caracteriza pelo acúmulo do Líquido cefalorraquidiano (LCR). O tratamento consiste em minimizar e favorecer uma qualidade de vida ao paciente. A doença apresenta múltiplos fatores, que pode está presente antes do nascimento ou alguns meses após sua vitalidade (URIBE et al, 2005). A forma mais comum do aparecimento da hidrocefalia está ligada ao cromossomo $\mathrm{X}$, que corresponde a $2,5 \%$ entre as causas de hidrocefalia congênita. Além disso, resulta no aumento do volume ventricular por causa de uma obstrução entre os locais de produção e absorção do líquido cérebroespinhal (LCE) sendo menos comum na produção excessiva (CALLEN, 200o).

Uma das causas mais frequente da hidrocefalia congênita, está relacionada ao quadro infeccioso da taxoplasmose e citomegalovírus, presente em caso de hidrocefalia fetal. Outra característica é a fronte alargada e os olhos desviados para baixo por causa do avanço suprapineal (PARTINGTON, 20oI).

As manifestações clínicas da doença podem variar conforme a idade, a evolução e os mecanismos de compensação da hipertensão intracraniana. No ínicio da lactância precoce, ocorre o aumento da cabeça, a fontanela anterior fica abaulada por causa da separação óssea do crânio, com o aumento da pressão intracraniana e excesso do LCR a cabeça fica tensa e não pulsátil, as veias do couro cabeludo ficam salientes e dilatadas, devidoá separação óssea que adelgaça a dilatação dos vasos, as suturas ficam separadas, ocorre perda constante dos cabelos e adelgaçamento dos ossos do crânio (FOSCHIERA, 2004).

No estado patológico da doença ocorre o sono profundo, do qual a criança pode ser acordar, podendo cair no sono novamente, a presença de reflexos nervosos é constante, a alteração do nível de consciência, contratura espástica da nuca, que faz com que o próprio corpo apresente estímulos diferentes (AMORIM, 2005). 
A anamnese bem detalhada é o principal avanço para investigação da doença. $\mathrm{Na}$ coleta de dados é necessário observar se a criança nasceu prematura, desenvolveu patologias associadas a mielomeningocele, meningite, encefalite da caxumba, etc. A fisioterapia é indicada no começo da doença, que irá ajuda no prognóstico, lembrando que as crianças portadoras deste tipo de enfermidade (hidrocefalia) podem ter um desenvolvimento lento, cujo tratamento fisioterápico ajudará na fase de crescimento, podendo obter o progresso mais saudável e próximo do normal. O tratamento para este tipo de patologia, é realizado com derivações dos ventrículos cerebrais para cavidade do peritônio. Essa drenagem se dar por uma válvula que anulam a hipertensão intracraniana da hidrocefalia (JUCÁ et.al., 2002).

A presença dos familiares é de fundamental importância neste momento, pois a adaptação da criança é de responsabilidade do coordenador, docente, AEE, enfermeiro e outros profissionais, já que os pais precisam de apoio e incentivo para se adaptar as dificuldades da criança e frente aos problemas que a mesma apresentar. Diante das dificuldades, os familiares devem receber apoio psicológico no reconhecimento e acompanhamento da doença (PIANNETTI, 200o). Bem como professores e coordenadores devem compreender aspectos de inclusão escolar. O ideal é que a escola tenha o atendimento educacional especializado (AEE) com profissionais neuropsicopedagogicos, psicopedagogos e que podem fazer a ponte entre o professor de sala regular, o próprio $\mathrm{AEE}$ e os pais dos alunos para acompanhamento com equipe de saúde multidisciplinar.

Vale ressaltar que este tipo de patologia é um problema constante a criança com hidrocefalia, e necessita ser avaliada diariamente. Lembrando que o acompanhamento da enfermeira reque cuidados minuciosos, tendo como importância o resultado dos exames de rotina, como tomografia, punção ventricular e punção lombar. Os profissionais da saúde e educação prestam cuidados, porque após a avaliação feita a criança, será mais fácil identificar os problemas e planejar uma assistência de qualidade (MASET, 2005).

O presente trabalho objetivou analisar a importância do coordenador e a hidrocefalia: um elo entre o atendimento educacional especializado, docentes, coordenadores e a equipe de saúde 


\section{FUNDAMENTAÇÃO TEÓRICA}

A assistência prestada ao portador de hidrocefalia deve estar fundamentada em princípios científicos, com objetivo prevenir falha no tratamento (CRUZ, 2005). O aumento do perímetro cefálico (PC) é mais frequente em criança com está patologia. O enfermeiro realiza a consulta de puericultura, tendo como objetivo observa a mensuração do $\mathrm{PC}$ e investigar algumas alterações cerebrais que possam ajuda na descoberta da doença atual (PRATES, 2005).

Ao nascimento, o PC mede em torno de I a $2 \mathrm{~cm}$ maior do que o torácico (MARCONDES, 2003). O cérebro do recém-nascido desenvolve rapidamente, cerca de 25 a $60 \mathrm{~cm}$ em apenas um ano, isso justifica o rápido aumento no PC, em relação a uma pessoa adulta. Para o controle do PC, o enfermeiro deve observar a fontanela anterior (Bregmática), a largura das suturas e a consistência do osso craniano. O fechamento da fontanela anterior ocorre em torno do roo ao $24^{\circ}$ mês de vida. $\mathrm{O}$ PC ao nascimento de um bebê a termo mede em média $35 \mathrm{~cm}$, podendo aumentar de acordo com a idade. O PC do pré-termo mede $33 \mathrm{~cm}$ ou menos, no entanto, deve leva em consideração um falso PC menor, devido ao cavalgamento ósseo nos primeiros dias após o nascimento. O enfermeiro deve realizar, a cada 2 dias, a mensuração do PC, onde é usado uma fita métrica, em volta do crânio, envolvendo os polos proeminentes (bossa occipital, atrás; as duas bossas frontais, frente) devendo ser feito até um ano de vida (SETTANNI, 2000).

$\mathrm{Na}$ observação do PC do pré-termo deve ser averiguado o gráfico de valor do PC, tendo como finalidade distinguir o aumento do crescimento da circunferência cefálica (CC), que ocorre nos portadores desta patologia.

O líquido cefalorraquidiano (LCR) é absorvido na cavidade abdominal quando se coloca um sistema de derivação ventrículo peritoneal (DVP) para minimizar a pressão intracraniana (PIC) em hidrocéfalos. No entanto, pode haver um aumento da quantidade de drenagem do (LCR), ou uma reabsorção inadequada devido a irritação crônica do peritônio, ocasionando uma peritonite subclínica (HENRIQUES, 2003).

$\mathrm{Na}$ ocorrência de distensão abdominal, é necessário impor uma restrição hídrica de dieta zero nas 24 horas, além de uma observação contínua, para evitar sobrecarga hídrica (DICCINI, 2006). O enfermeiro observa e mensura o peritônio 
torácico (PT), que deve ser relacionado com o PC. A região abdominal do RN, em decúbito dorsal, fica aproximadamente ao mesmo nível do tórax. Ao mensurar o PT, a fita métrica deve passar em torno do tórax da criança, que deve estar em posição de dorso flexão e flexão dos joelhos para que a região abdominal esteja bem relaxada (KLIEMANN,

$\mathrm{O}$ aumento da PIC é uma síndrome que prejudica muitos pacientes com distúrbios neurológicos, devido as condições patológicas que afeta a relação entre o volume de líquido e a pressão intracraniana (SMELTZER, 2006).

O conhecimento da PIC é importante, pois permite ao profissional intervir, ajustando a terapêutica em caso de necessidade, em decorrência de complicação precoce (SPAHO, 2006). O controle da PIC, é também uma responsabilidade do enfermeiro, incluindo a preparação de materiais e a avaliação do paciente. Em situações fisiológicas, a PIC se mantém abaixo de $15 \mathrm{mmHg}$ em adulto, de $7 \mathrm{mmHg}$ em criança, e acima de $20 \mathrm{mmHg}$ em patologias e lesões temporárias (GRACÍA et al, 2006).

O monitoramento da PIC é realizado através de procedimentos invasivos, dentre esses está a derivação ventricular externa (DVE), onde é introduzido um cateter com um sensor intracraniano, que é adaptado a um monitor para emissão de ondas e valores numéricos que descrevem a situação da PIC. O posicionamento do paciente é de extrema importância, devendo o mesmo ser mantido com a cabeceira elevada a 30ㅜ, ficando a cabeça mantida em linha média, pois qualquer elevação ou movimento desnecessário poderá levar ao aumento ou diminuição da PIC, resultando em valores não reais (GRACÍA et al; 2006).

O cuidado da equipe de educação e enfermagem ao paciente com a permanência do DVE é essencial, pois o paciente tem que ser acompanhado e monitorado frequentemente (SPAHO, 2006).

Medidas simples, após a realização de determinados procedimentos, são de extrema importância para evitar complicações, como: higiene das mãos com sabão antisséptico e álcool a 70\% antes e após o manuseio do paciente; a higiene do couro cabeludo deve ser realizada a seco a cada 48 horas, com álcool a 70\%, não devendo molhar o curativo; antes de movimentar o paciente (mudança de decúbito, mudança 
da altura da cabeça, movimento da altura da bolsa de drenagem) deve-se pinçar o sistema de derivação por no mínimo 30 minutos; tratando-se de um circuito fechado, é essencial vigilância nas conexões, observando quando houver desconexão do mesmo, pinçando o local próximo ao paciente, fazer o procedimento de forma asséptica e comunicar ao neurocirurgião; a posição da cabeça do mesmo deve estar à $30^{\circ}$; observar se a bolsa de drenagem esta na altura indicada ( $10 \mathrm{~cm}$ ), tomando como referência o condutor auditivo externo. Caso a bolsa coletora esteja na posição abaixo do ponto indicado, ocorrerá o aumento da drenagem do líquor presente no reservatório ventricular (SPAHO, 2007).

A observação frequente dos sinais vitais, nível de consciência, reflexo motores e pupilares, são indispensável para avaliação de possíveis complicações pósoperatórias. Até que a criança se levante deve ser observado a cada 15 minutos e os sinais vitais de ra 6 horas, dependendo das condições que o mesmo se encontre, pois um aumento da pressão arterial sistólica indica aumento da PIC e isquemia cerebral, dificultando assim o tratamento. É importante observar o ritmo respiratório, pois, pode ocasionar o aumento da massa intracraniana. Já o aumento da temperatura corporal pode ser sugestivo de um quadro infeccioso. A avaliação do exame laboratorial é importante, pois permite que o enfermeiro consiga avaliar complicações recentes: queda de hematócrito no pós-operatório imediato, por exemplo, resulta em hemorragia intraoperatória. As pressões dos gases sanguíneos devem ser avaliadas através da gasometria arterial, que investiga o valor da $\mathrm{PCO}_{2}$, que quando muito alto induz a vasodilatação, provocando o aumento da PIC (GRILLO, 2003).

A qualidade da assistência depende muito do desempenho da equipe multiprofissional (médicos, fisioterapeutas, psicopedagogos, neuropsicopedagogos e etc), no entanto, é a enfermagem que irá executar o plano de cuidados e observar o paciente intensamente, procurando ajuda-lo, instruindo e orientando a família quanto à ação esperada do medicamento. Algumas orientações devem ser fornecidas durante o tratamento da hidrocefalia, relacionado ao uso de diurético, a importância da ingestão de líquidos, dieta rica em potássio (tomate, banana) para reduzir a hipotensão postural durante o tratamento, a mudança de decúbito, sendo a mesma lentamente; o uso de protetor solar e vestimentas adequadas para evitar a fotossensibilidade; os 
lábios secos podem ser reduzidos com uso de lascas de gelo; permanecer em repouso absoluto para evitar tontura; observar rigorosamente balanço hídrico e controlar o peso constantemente, a função hepática, a glicemia capilar (em criança diabética) e a hipocalemia (fraqueza e câimbras musculares) (AME, 2006).

Por meio da consulta de enfermagem, realiza-se a busca dos dados através do histórico do paciente, onde as identificações que correspondem aos diagnósticos de enfermagem levam a perceber o grau de dependência do paciente (CRUZ, 2008).

O coordenador, docente, AEE, enfermeiro e outros profissionais deve conhecer as estratégias de educação a saúde, além de promover uma assistência integral e plena a criança com hidrocéfalo, fortalecendo sua confiança com a família, que contribui para compreensão e convivência do portador da doença (SOUZA, 2009). Sendo assim, o objetivo desta revisão foi realizar uma abordagem sobre a criança portadora de hidrocefalia, seus sinais e sintomas clínicos e a atuação do coordenador, professor e equipe de saúde no processo da assistência ao paciente.

\section{METODOLOGIA}

Trata-se de uma revisão da literatura, cuja coleta de dados foi efetuada por meio de um levantamento bibliográfico nas bases de dados LILACS, MEDLINE e SCIELO, no período de 2000 a 2010. Os descritores utilizados foram: hidrocefalia, coordenador, docente, escola, pediátrico e cuidados de enfermagem. A pergunta norteadora foi: quais as ações do coordenador, docente, AEE, enfermeiro e outros profissionais a criança portadora de hidrocefalia descrita na literatura científica. Foram considerados elegíveis, os artigos que respondessem à pergunta norteadora.

\section{CONCLUSÃO}

A hidrocefalia é uma patologia que afeta a criança por toda sua vida. Afeta o processo da relação com a família, com a coordenação, os pais, o AEE e a equipe de enfermagem. A assistência de qualidade e humanizada, deve ser favorecida a está criança ao longo de sua vida, cabendo ao coordenador, docente, AEE, enfermeiro e outros profissionais implantar estratégias que venham melhorar o quadro da doença, promovendo saúde ao seu contexto biopsicossocial e espiritual. O portador de 
hidrocefalia tem um prognóstico desfavorável à criança com necessidade de um tratamento intensivo e controle das complicações. Sendo assim, a equipe de educação e de enfermagem deve proporcionar um ambiente tranquilo, acolhedor, por meio de uma assistência centrada na família.

Desta forma é importante estimular a pesquisa de enfermagem sobre esta temática, fortalecendo sua prática baseada em evidências, para o planejamento de cuidados específicos e individualizados ao portador da doença.

\section{REFERÊNCIAS}

MASET, A. L.; CAMILO J. R.; VIEIRA, E. D. R. Considerações hidrodinâmicas sobre a derivação liquórica - Parte II AMORIM, R. C.; OLIVEIRA E. M. O ensino e práticas de cuidado: o caso de um curso de graduação em enfermagem. Acta Paul Enferm. v.18 (I), p. 25-30, 2005 .

AME. Dicionário de administração de medicamentos na enfermagem. $5^{\underline{a}}$ ed. Rio de Janeiro, EPUB, p. 922, 2006.

AQUINO, H. B. Complicações abdominais do cateter distal no tratamento da hidrocefalia com migração, perfuração e/ou extrusão:uma hipótese inflamatória. [Dissertação]. Campinas: Universidade Estadual de Campinas, 2004.

CRUZ, D. A. L. M. Processo de enfermagem e classificações. In: GAIDZINSKI, R. R.; SOARES, A. V. N.; LIMA, A. F. C.; GUTIERREZ, B.A.O.; CRUZ, D. A. L. M; REGENSKI, N. M. B.; SANCINETTI, T.R. Diagnóstico de enfermagem na prática clínica. Porto Alegre: Artmed, 2008.

CALLEN, P. W. Ultrasonography in obstretrics and Gineology. $4^{\underline{a}}$ ed. W. B. Saunders Company: Philadelphia, 2000.

CRUZ, D.; PIMENTA, C. Prática baseada em evidências, aplicada ao raciocínio diagnóstico. Rev. Latino Americana Enfermagem, v. 13 (3), p.415-22, maio-junho, 2005. 
DICCINI, S. Assistência de enfermagem em pacientes neurocirúrgicos. In: BRAGA, F. M.; MELO, P. M. P. Neurocirurgia: Série Guias de Medicina Ambulatorial e Hospitalar. São Paulo: Manole, 2006.

FOSCHIERA, F.; VIERA, C. S. O diagnóstico de enfermagem no contexto das ações de enfermagem: percepção dos enfermeiros docentes e assistenciais. Revista Eletrônica de Enfermagem, v. 6 (o2), p.189-198, 2004.

GRILLO, E.; SILVA, R. J. M. Defeitos do tubo neural e hidrocefalia congênita. Por que conhecer suas prevalências? J. Pediatr., v. 79 (2), 2003.

GARCÍA, J. Actuación de enfermería en la monitorización de la presión intracraneal (P.I.C.). Enfermería Integral, p. 40-44, jun. 2006.

HENRIQUES, J. G. B.; PINHO, A. S.; PIANETTI, G. Complicação de derivação ventrículo-peritoneal: hérnia inguinal com migração do cateter para o saco escrotal. Relato de caso. Arquivo de Neuropsiquiatria, São Paulo, v. 6I (2), p. 486-489, jun. 2003. JUCÁ, et al. Tratamento de hidrocefalia com derivação ventrículo-peritoneal: análise de 150 casos consecutivos no Hospital das Clínicas de Ribeirão Preto. Acta Cir. Bras., v.17 (3), São Paulo, 2002.

MARCONDES, E. Pediatria básica. 8ª Ed. São Paulo: Sarvier, p.1991. 1789 CONDES, E.; SETIAN, N.; CARRAZZA, ALVES, E. R. S.; JAQUES, A. E.; BALDISSERA, V. D. A.: O efeito sifão em sistemas de drenagem externa. Arq Bras Neurocir., v. 24 (2) p. 45-51, 2005.

PARTINGTON, M. D. Congenital hidrocephalus. Neurosurng Clin. N. Am.; 36: 737742, 200I.

PIANETTI FILHO, G. Válvulas em Neurocirurgia. In: POHL, F. F; PETROIANU, A. Tubos, sondas e drenos. Rio de Janeiro: Guanabara Koogan, 2000.

PRATES, M. A.; ZANON-COLLANGE, N. Hidrocefalia. In: BRAGA, F. M.; MELO, P. M P. Neurocirurgia: Série Guias de Medicina Ambulatorial e Hospitalar. São Paulo: Manole, 2005.

KLIEMANN, S. E.; ROSEMBERG, S. Hidrocefalia derivada na infância: um estudo clínico epidemiológico de 243 observações consecutivas. Arq. Neuropsiquiatr., v. 63 (2b), 2005. Disponível em: www.scielo.br. Acesso em: os de junho de 2006. 
RAMOS, et al. Pediatria neonatal. In: MARCONDES, E. et al. Pediatria básica: pediatria geral e neonatal. $9^{\text {a }}$ ed. São Paulo: Sarvier, p. 843, cap. 5, 2003.

SMELTZER, S. C.; BARE, B. G. Brunner e Suddarth: tratado de enfermagem médico cirúrgica. Io ${ }^{-}$ed. Rio de Janeiro: Guanabara Koogan, p. 2419, 2006.

SETTANNI, F. Drenagens em Neurocirurgia. In: POHL, F. F; PETROIANU, A. Tubos, sondas e drenos. Rio de Janeiro: Guanabara Koogan, 2000.

SPAHO, N. et al. Guias de práctica clínica para el manejo del drenaje ventricular externo. Revista Argentina Neurocirurgia, Buenos Aires, v. 20, p.I43-I46, 2006.

URIBE, R. C. L. et al. Hidrocefalia ligada al cromosoma X. Reporte de un caso. Revista Mexicana de Pediatria, v. 72 (6), p. 301-304, nov. 2005. 